\title{
Binding Sites of Drosophila melanogaster Sex Peptide Pheromones
}

\author{
Michael Ottiger, ${ }^{1}$ Matthias Soller, ${ }^{2}$ Reinhard F. Stocker, ${ }^{3}$ and Eric Kubli ${ }^{1}$ \\ ${ }^{1}$ Zoologisches Institut Universität Zürich-Irchel, Winterthurerstrasse 190, CH-8057 Zürich, \\ Switzerland \\ ${ }^{2}$ Department of Biology and Center for Complex Systems, Brandeis University, Waltham, \\ Massachusetts 02154 \\ ${ }^{3}$ Department of Biology and Program in Neuroscience, Université de Fribourg, Pérolles, $\mathrm{CH}-1700$ \\ Fribourg, Switzerland
}

Received 19 January 2000; accepted 21 March 2000

\begin{abstract}
Drosophila melanogaster sex peptide (SP) and Ductus ejaculatorius peptide (DUP99B) are male pheromones transferred in the seminal fluid to the female during copulation. Both reduce sexual receptivity and stimulate oviposition in females. The presence of high-affinity SP and DUP99B binding sites in the female were investigated by incubation of cryostat tissue sections with ${ }^{125}$ I-iodinated peptides and subsequent autoradiography. We found that in adult females radiolabeled SP and DUP99B bind to peripheral nerves, the subesophageal ganglion, the cervical connective, to discrete parts of the thoracic ganglion, and to the genital tract. Weak and uniform labeling was detected in the neuropil of the brain and the thoracic ganglion. The
\end{abstract}

labeling pattern in the nervous system suggests binding of the peptides to sensory afferents or glial cells. Scatchard analysis of the binding of ${ }^{125}$ I-DUP99B to antennal nerves yielded a dissociation constant $K_{\mathrm{d}}$ of $6.4 \mathrm{n} M$. Competition experiments with peptide fragments show that the peptides bind with their homologous $\mathrm{C}$-terminal regions. Binding sites in the nervous system of females are established throughout sexual maturation. Prominent binding of the peptides to afferent nerves suggests modification of sensory input. ๑ 2000 John Wiley \& Sons, Inc. J Neurobiol 44: 57-71, 2000

Keywords: Drosophila melanogaster; nervous system; sex peptides; receptor; reproduction
In many insect females, mating elicits a series of well-defined behavioral changes (Gillott, 1988; Eberhard, 1996). Among the most conspicuous are (1) an increase in egg-laying rate and (2) the reduction of receptivity (i.e., a refractory behavior toward courting males). These changes in female reproductive behav-

Correspondence to: E. Kubli (ekubli@zool.unizh.ch).

Contract grant sponsor: Swiss National Science Foundation.

Contract grant sponsor: Pro Scientia.

Contract grant sponsor: the Hescheler-Stiftung.

Contract grant sponsor: the Julius Klaus-Stiftung.

(C) 2000 John Wiley \& Sons, Inc. ior are induced by substances synthesized in the male genital tract and transferred as part of the seminal fluid to the female during copulation (Kubli, 1996; Wolfner, 1997). Hence, these molecules qualify as sex pheromones as defined by Karlson and Lüscher (1959).

Three peptide pheromones have been isolated from Drosophila melanogaster males, affecting one or both of the above-mentioned postmating responses of the female. Sex-peptide (SP; Chen et al., 1988) and the recently isolated DUP99B (Ductus ejaculatorius peptide, cytological localization 99B; Saudan, 1993; Hauck, 1995; Swiss Prot no. P81160) elicit both post- 
A

sSP $_{6-20} Y$
SSP $_{17-36}$
YsSP
sSP
sDup99B
YsDup99B5-17

NRKPTKFPIPSPNPRY PNPRDKWCRLNLGPAWGGRC

YWEWPWNRK PTKF I PSPNPRDKWCRLNLGPAWGGRC

${ }^{\star}$ WEWPWNRKPTKFPIPSPNPRDKWCRLNLGPAWGGRC

QDRNDTEW-IQSQKDREKWCRLNLGPYLGGRC Y DTEW - IQSQKDREK

$"$

B

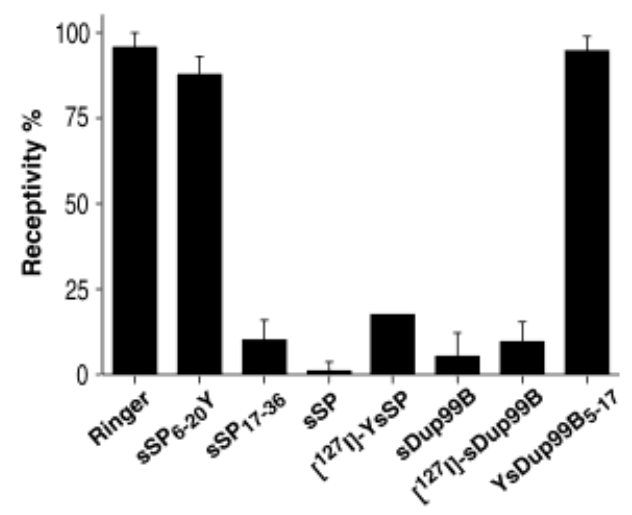

C

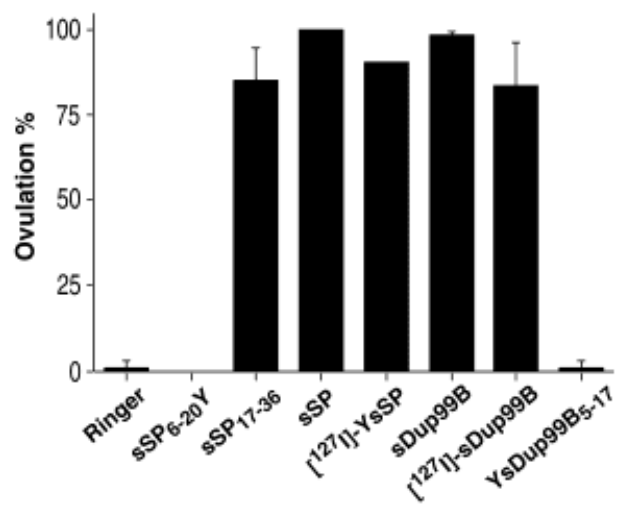

Figure 1 Peptide sequences and biological activity of synthetic peptides, fragments thereof, and nonradioactively iodinated derivatives. (A) Sequences of synthetic sex peptide (sSP), synthetic DUP99B (sDUP99B), and fragments thereof. The tyrosine residues ( $\underline{Y})$ at the N-terminal ends of YsSP and $\mathrm{YsDUP} \mathrm{B}_{5-17}$, and at the C-terminal end of $\mathrm{sSP}_{6-20} \mathrm{Y}$, respectively, have been introduced during peptide synthesis for iodination purposes. Identical amino acids are indicated by strong shading, similar amino acids by weak shading. (B) Ovulation after injection of synthetic peptides and fragments thereof, nonradioactively iodinated derivatives, and Ringer's solution as a control. Twenty virgin females were used for one experiment. The mean values \pm S.D. calculated from three experiments are shown (two experiments for ${ }^{127} \mathrm{I}-\mathrm{YsSP}$ ). Both full-size peptides elicit ovulation independent of the presence of ${ }^{127}$ I. Note that the C-terminal fragment of SP is sufficient to induce ovulation, whereas the N-terminal fragments of sDUP99B and sSP, YsDUP99B ${ }_{5-17}$ and $\mathrm{SSP}_{6-20} \mathrm{Y}$, do not induce ovulation, nor does Ringer injection. (C) Receptivity after injection of the same peptides as in (B). Thirty virgin females were used for one experiment. The mean values \pm S.D. calculated from three experiments are shown (two experiments for ${ }^{127} \mathrm{I}-\mathrm{YsSP}$ ). All peptides eliciting ovulation also reduce receptivity. Note that only peptides containing the C-terminal amino acids (encoded by the second exon) are active. Addition of nonradioactive iodine $\left({ }^{127} \mathrm{I}\right)$ does not affect the biological activity.

mating responses when injected into virgin females and are synthesized in the accessory glands and the Ductus ejaculatorius, respectively. The peptide Acp26Aa is synthesized in the male accessory glands and stimulates oviposition during the first day after copulation (Herndon and Wolfner, 1995).

SP and DUP99B show strong homologies in their $\mathrm{C}$-terminal regions, encoded by the second exons of their respective genes (Fig. 1; Hauck, 1995). These parts of the two peptides are essential for eliciting increased egg laying rate and reduced receptivity (Chen et al., 1988; Schmidt et al., 1993; Hauck, 1995). On the basis of their sequence homology in the second exon, we consider them as the founding members of the SP pheromone family. Whether they are functionally redundant in vivo with respect to these two postmating responses, however, is not known.

Only indirect evidence is available regarding the molecular nature of putative SP receptors and their distribution in the female. Analysis of structure-function relationships has shown that the $\mathrm{C}$-terminal part of SP is essential to elicit oviposition and reduced receptivity (Schmidt et al., 1993). SP fragments elicit either both or none of the postmating responses. Furthermore, ovulation, oviposition, and reduction of receptivity are induced at the same critical concentra- 
tion of 0.6 pmol SP/female (Kubli, 1996; Schmidt et al., 1993). Both findings suggest that a common receptor is present at the top of the SP response cascade. This view is also supported by results obtained by Nakayama et al. (1997), with membrane-bound SP fusion proteins expressed in various tissues of transgenic enhancer-trap lines. Their experiments show that either both responses are elicited or none and that an active response is correlated with expression of the membrane-bound SP fusion protein in the head.

$\mathrm{SP}$ is the best characterized male peptide sex pheromone influencing female reproductive behavior in insects (Kubli, 1996). Besides affecting oviposition and receptivity, SP also activates egg production. Vitellogenic oocyte progression as well as ovarian yolk protein synthesis and uptake into the ovary are stimulated (Soller et al., 1997, 1999). The juvenile hormone $(\mathrm{JH})$ analogue methoprene mimics SP-mediated stimulation of vitellogenic oocyte progression in sexually mature, virgin females (Soller et al., 1999). Because addition of SP to the corpora allata (CA) in vitro stimulates JH synthesis (Moshitzky et al., 1996), JH is very likely a downstream component in the SP response cascade, and the CA a target for SP. The JH analogue, however, neither elicits increased oviposition nor reduces receptivity. Hence, SP must have additional targets with separate signal cascades leading to the two postmating responses (Soller et al., 1999). These latter effects are very likely mediated via the nervous system.

It has been shown that the memory and learning mutant dunce does not respond to SP (Chapman et al., 1996). The dunce gene encodes a cAMP phosphodiesterase (Chen et al., 1986). This enzyme is expressed in many parts of the adult fly, but mainly in the mushroom bodies (Nighorn et al., 1991). Chemical ablation of the mushroom bodies, however, does not affect the SP responses (Fleischmann, 1997), suggesting that these brain structures are not involved in the SP response cascade.

As shown by gynandromorph experiments, some cells of the dorsal-anterior region of the brain must be female to allow receptive behavior in females (Tompkins and Hall, 1983). Furthermore, in females with a cauterized pars intercerebralis, a neuroendocrine center of the insect brain, mating no longer stimulates oviposition (Boulétreau-Merle, 1976). Because the genital tract is the first part of the female to encounter SP pheromones, it might also be a target for the two peptides. Taken together, parts of the nervous system, the CA, and the genital tract have to be considered as putative targets for SP. In this article we investigate the binding sites of ${ }^{125}$ I-iodinated SP and DUP99B on tissue sections of adult flies, a method successfully used in pharmacology to localize peptide and other receptors (e.g., Lichtensteiger et al., 1996). We show that only biologically active peptides label specific parts of the nervous system and the genital tract of females. Strong labeling was found in the proximal parts of all afferent nerves, established during sexual maturation of the adult female. Hence, the localization of putative receptors at these sites might indicate a modification of sensory input by sex peptide pheromones, resulting in a different behavioral response to the same stimuli before and after mating.

\section{MATERIALS AND METHODS}

\section{Fly Stocks and Experimental Animals}

Flies were kept on standard cornmeal-agar food in a 12-h/ 12-h light/dark cycle. To produce large numbers of virgin females, Oregon R wild-type males were crossed to females with the genotype $y \mathrm{~cm} \mathrm{Sxl} l^{M I}$; $\operatorname{vir}^{f l} b w / S M 5$ and females lacking the SM5 balancer were used (genotype of experimental females: $y \mathrm{~cm} \mathrm{Sxl} l^{M I} /+$; $v i r^{f l} b w /+$; Hilfiker, 1990; Soller et al., 1999). At the day of eclosion, virgin females were collected on ice, and 70-80 females were kept in bottles at $25^{\circ} \mathrm{C}$ for 5 days with one food change the day before the experiment. Wild-type males were separated from females after eclosion and kept under identical conditions. As a control, all experiments were also done with Oregon R wild-type virgin females. No differences were detected between experimental and control females (data not shown).

\section{Peptides and Biological Tests}

All peptides used were synthetic and were synthesized according to Schmidt et al. (1993) or obtained from Neosystem (Strasbourg, France). For labeling purposes, tyrosine residues were introduced into synthetic peptides at either the $\mathrm{N}$ - or C-terminus. Peptides were dissolved in water, lyophilized, and stored at $-80^{\circ} \mathrm{C}$. Peptides used for injection experiments were either dissolved in water or in Ringer's solution, and $50 \mathrm{~nL}$ containing $3 \mathrm{pmol}$ of peptide were injected as described (Soller et al., 1997). Receptivity was tested by confronting 3 females with 7 inexperienced Oregon $\mathrm{R}$ wild-type males in one vial $4-5 \mathrm{~h}$ after injection. From a total of 30 females, receptivity was calculated as percentage of females mating within $1 \mathrm{~h}$. Ovulation was tested by anesthesizing females with $\mathrm{CO}_{2} 4-5 \mathrm{~h}$ after injection and expressed as the percentage of females extruding an egg from a total of 20 females. 


\section{lodination and Purification of lodinated Peptides}

RP-HPLC-purified peptides (Schmidt et al., 1993) were

${ }^{125}$ I-iodinated with Iodo-Beads according to the manufacturer's protocol (Pierce, Rockford, IL) on tyrosine residues. Briefly, $5.4 \mathrm{nmol}$ peptide was incubated with $0.8 \mathrm{mCi}$ ${ }^{125} \mathrm{I}-\mathrm{Na}$ (Amersham, Arlington Heights, IL) and 2 IodoBeads in $250 \mu \mathrm{L}$ reaction buffer (100 $\mathrm{m} M$ phosphate, $\mathrm{pH} 8$ ). The reaction was terminated by removing the Iodo-Beads from the reaction after $15 \mathrm{~min}$. Labeled peptides were then purified by RP-HPLC on a Vydac (Hesperia, CA) C18 Protein \& Peptide $(250 \times 2 \mathrm{~mm})$ column. Specific activity of labeled peptides was calculated after measuring the peptide concentration spectrophotometrically and incorporated radioactivity with a scintillation counter (LS 3801; Beckman, Berkeley, CA) and was found to be higher than 1600 $\mathrm{Ci} / \mathrm{mmol}$. The purity of the probes was further checked by running aliquots on 17\% Tricine-gels (Schägger and von Jagow, 1987) with subsequent autoradiography. Nonradioactive labeling of peptides was done by substituting radioactive ${ }^{125} \mathrm{I}$ with NaI to a final concentration of $150 \mu \mathrm{M}$. Iodination products were then separated by RP-HPLC and analyzed by mass spectroscopy (LC-MC; MS-Pesci Ex Api III Biomolecular Mass Analyzer, Beaverton, OR).

\section{Cryostat Tissue Sections and Autoradiography}

For cryostat sections flies were embedded in Tissue Freezing Medium (Jung) and cut on a Leica SC3000 cryostat at a thickness of $20 \mu \mathrm{m}$. Whole flies were sectioned in rubber blocks (Leica AG, Deerfield, IL), and head sections were prepared by fitting 15 flies into a "collar," allowing simultaneous cutting of all heads (Heisenberg and Böhl, 1979). Subbed slides were prepared as in Hafen and Levine (1986). Binding assays were done according to Dudai and Amsterdam (1977) and Lichtensteiger et al. (1996) with some modifications. Frozen sections were allowed to thaw for 20 min at room temperature before preincubation with $50 \mathrm{mM}$ Tris- $\mathrm{HCl}(\mathrm{pH} 7.4)$ and $0.4 \%(\mathrm{w} / \mathrm{v})$ BSA for $20 \mathrm{~min}$. Slides were then air-dried for $2 \mathrm{~h}$. Incubations with labeled peptides were done in $150 \mu \mathrm{L} 50 \mathrm{~m} M$ Tris- $\mathrm{HCl}$ (pH 7.4), $0.4 \%$ (w/v) BSA, and $120 \mathrm{mM} \mathrm{NaCl}$ at room temperature for $1 \mathrm{~h}$ under a coverslip using final concentrations of 0.72-72 $\mathrm{n} M$ ${ }^{125}$ I-DUP99B or $3-36 \mathrm{n} M{ }^{125} \mathrm{I}$-YSP, respectively. Competition experiments were done by adding a 250 -fold excess of unlabeled peptide. Incubations were stopped by washing the slides $4 \times$ for $1 \mathrm{~min}$ in $50 \mathrm{mM}$ Tris- $\mathrm{HCl}$ ( $\mathrm{pH} 7.4$ ), followed by $2 \times 10$-s washes in double distilled water. Slides were then dried overnight at room temperature. Specificity of binding was also analyzed using $0.4 \%$ (w/v) ovalbumin as blocking agent.

For autoradiography, sections were covered with photo emulsion LM1 (Amersham) according to the manufacturer's instructions, dried at room temperature over night, and exposed for 5-30 days. To avoid cross-exposure, lead plate- lets were placed between individual slides. After exposure slides were developed in a fivefold dilution of Ilford Phenisol developer at $18^{\circ} \mathrm{C}$, washed for $1 \mathrm{~min}$ in tap water, fixed in fourfold diluted Ilford Paper Fixer, washed again 5 min in tap water, rinsed in double distilled water, and air-dried.

The dissociation constant $K_{\mathrm{d}}$ was determined by incubation of frontal head sections with increasing concentrations of labeled peptide and subsequent autoradiography. After autoradiography the signal intensities on the antennal nerves (minus background) were determined using NIH-image program (http://rsb.info.gov/nih-image/) on a Macintosh computer (Apple Computer, Cupertino, CA).

\section{RESULTS}

\section{Probes for Autoradiography and Their Biological Activity}

Synthetic SP and synthetic DUP99B (sSP and sDUP99B; $\mathrm{s}=$ synthetic. $\mathrm{SP}$ and DUP99B $=$ native peptides) and fragments thereof [Fig. 1(A)] were modified with iodine at tyrosine residues. Because native SP does not contain tyrosine (Y), a tyrosine residue was added at its $\mathrm{N}$-terminus during peptide synthesis. After iodination, the peptides were purified by HPLC and analyzed by mass spectroscopy.

Sex peptide and DUP99B elicit both postmating responses (Saudan, 1993; Schmidt et al., 1993). Because the iodinated, synthetic peptides differ from the native peptides [addition of iodine in both peptides, addition of Y in SP; Fig. 1(A)], their biological activities were tested before and after iodination with nonradioactive iodine $\left({ }^{127} \mathrm{I}\right)$. All peptides containing the amino acids encoded by the second exons were active, irrespective of the presence or absence of ${ }^{127} \mathrm{I}$ [Fig. 1(B,C)]. The peptides $\mathrm{sSP}_{6-20} \mathrm{Y}$ and $\mathrm{YsDUP} 9 \mathrm{~B}_{5-17}$ were inactive in all respects.

\section{Competition Experiments: SP and DUP99B Bind to the Same Sites}

The specificity of binding for each peptide was investigated in competition experiments involving an excess of unlabeled peptides or fragments thereof [Fig. 1(A)]. As explained below, radioactive ${ }^{125}$ I-iodinated sSPs bind to specific tissues of the female. To demonstrate the specificity of binding, we chose frontal head sections, because the antennal nerve shows strong labeling in a low-background environment [Fig. 2(A)]. The strong labeling of the antennal nerve also allows the determination of the dissociation constant $K_{\mathrm{d}}$.

Competition of ${ }^{125}$ I-iodinated sDUP99B with a 

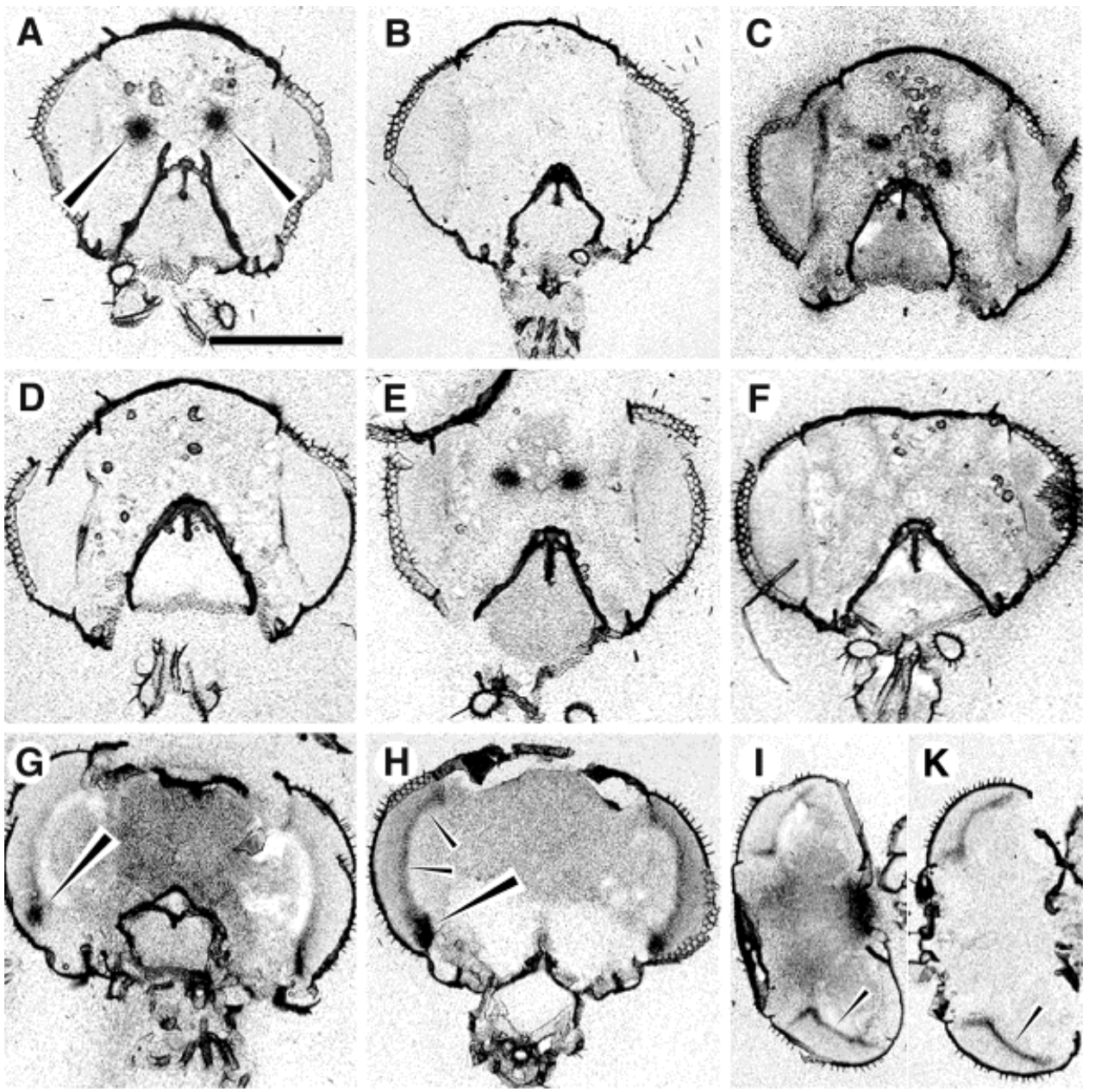

Figure 2 sDUP99B and sSP bind to the same sites at the antennal nerve with their homologous C-terminal parts. (A-H) Frontal head sections; (I,K) horizontal sections through heads of 5-day-old, sexually mature virgin $D$. melanogaster females. (A) Binding of ${ }^{125}$ I-sDUP99B to the antennal nerve (arrowheads). (B) Competition of ${ }^{125}$ I-sDUP99B binding with a 250-fold excess of sDUP99B. (C) Binding of ${ }^{125} \mathrm{I}-$ YsSP to the antennal nerve. (D) Competition of ${ }^{125} \mathrm{I}-\mathrm{sDUP} 99 \mathrm{~B}$ binding with a 250 -fold excess of sSP. (E) ${ }^{125} \mathrm{I}$-sDUP99B binding is not competed with a 250 -fold excess of YsDUP99B $_{5-17}$. (F) Competition of ${ }^{125}{ }^{2}$-YsSP binding with a 250 -fold excess of $\mathrm{sSP}_{17-36}$. (G) Binding of ${ }^{125}$ I-sDUP99B in the brain and along the optical tracts (arrowhead). (H) A 250-fold excess of sDUP99B competes ${ }^{125}$ I-sDUP99B binding only in the brain, whereas unspecific labeling remains at the optical tracts (arrowheads). (I,K) Same experiment with horizontal sections as in $(\mathrm{G})$ and $(\mathrm{H})$. Note the strong labeling of the cervical connective in (I) but not in the competition experiment in (K), whereas unspecific labeling of the optical tracts remains unaltered (arrowheads). Scale bar $=300 \mu \mathrm{m}$.

250-fold excess of unlabeled sDUP99B or sSP eliminates binding to the antennal nerve [Fig. 2(A, B,D)]. Also, ${ }^{125}$ I-YsSP can be competed with unlabeled sDUP99B (data not shown). The biologically active fragment $\mathrm{SSP}_{17-36}$ [corresponding to the C-terminus of SP, Fig. 1(A)] competes with labeled sSP [Fig. 2(F)]. The biologically inactive fragments $\mathrm{YsDUP} 9 \mathrm{~B}_{5-17}$ and $\mathrm{sSP}_{6-20} \mathrm{Y}$ [Fig. 1(A)], however, cannot displace the full-size, labeled sDUP99B [Fig. 2(E), and results not shown]. Binding of ${ }^{125}$ I-YsSP yielded the same pattern as ${ }^{125}$ I-sDUP99B [Fig. 2(C)].

The same results were obtained in all other parts of the fly (i.e., the C-terminal parts of the peptides are necessary for binding) and binding of full-length peptides can not be competed with $\mathrm{N}$-terminal fragments. Because labeling with sDUP99B resulted in a better signal-to-background ratio [cf. Fig. 2(A,C)], all results presented below are based on experiments with 


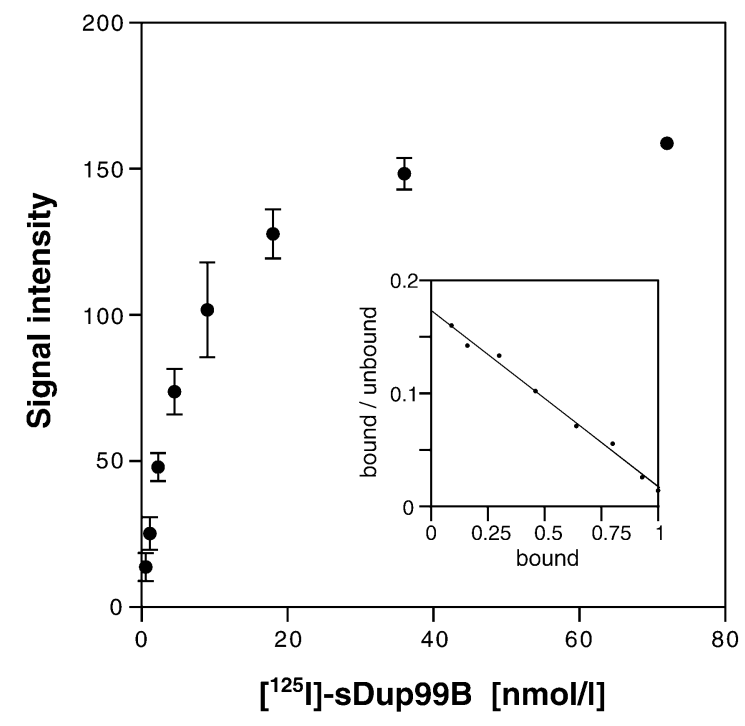

Figure 3 Determination of the dissociation constant $K_{\mathrm{d}}$. The saturation curve for the binding of ${ }^{125} \mathrm{I}$-sDUP99B to the antennal nerve in frontal sections through the head of 5-dayold, sexually mature virgin $D$. melanogaster females is shown. Inset: Scatchard plot of averaged data from saturation curve. $K_{\mathrm{d}}$ was determined as $6.4 \times 10^{-9} M$. The signal intensities of at least 12 autoradiographs of antennal nerves per ${ }^{125}$ I-sDUP99B concentration were measured using a NIH-image program on a Macintosh computer. Signal intensities are reported in calibrated units (average gray value of a circular selection within the antennal nerve minus the average gray value of the same size selection outside the antennal nerve $=$ background value).

${ }^{125}$ I-sDUP99B. Where not explicitly mentioned, however, radiolabeled YsSP yielded the same results. Unspecific binding, which could not be competed, was found only in the optical tracts [Fig. 2(G-K), and see later]. Taken together, these results show that both peptides recognize the same binding sites and that the C-termini, showing strong homology, are the relevant parts for binding.

\section{Determination of the Dissociation Constant}

To characterize the binding of sDUP99B to tissue sections, we determined the dissociation constant $K_{\mathrm{d}}$. Cryostat tissue sections of the head were incubated with a dilution series of radioactive probe, and the signal intensities on the antennal nerve were measured after autoradiography. The saturation curve was transformed into a Scatchard-plot and the dissociation constant $K_{\mathrm{d}}$ determined as $6.4 \times 10^{-9} M$ (Fig. 3).

\section{Sex-Peptide Pheromones Label Specific Parts of the Female Nervous System and the Genital Tract}

All results reported below were obtained with the same procedure, that is, ${ }^{125} \mathrm{I}$-sDUP99B was incubated on cryostat tissue sections, and the slides were subsequently autoradiographed. Incubation of tissue sections of sexually mature virgin females with ${ }^{125} \mathrm{I}-$ sDUP99B labeled specific parts of the nervous system and the genital tract (Figs. 4 and 5).

Incubations of frontal sections of the head [Fig. 4(A)] resulted in strong labeling of the antennal nerve [Fig. 4(B,C)], pharyngeal nerve [Fig. 4(C)], the antennal mechanosensory center [Fig. 4(D)], the periphery of the subesophageal ganglion with branching labial nerve [Fig. 4(E)], and the cervical connective [Fig. 4(F)]. Weak labeling was found in the brain [Fig. 4(D,E)] and very weak labeling in the antennal lobes [Fig. 4(D)].

Figure 5 shows an overall view of the labeling of the central nervous system (CNS), the peripheral nervous system, and the genital tract in horizontal, vertical, and oblique sections. Prominent and specific labeling was found in the CNS, along different peripheral nerves, the oviduct, and in the uterus. Specifically, in the head the antennal [Fig. 5(D)], labial [Fig. 5(E,K)], and the accessory pharyngeal nerves were labeled [Fig. 5(E)]; in the thorax, the leg [Fig. 5(D,E,G,H,K)], wing [Fig. 5(B,C,G,H)], haltere [Fig. $5(\mathrm{~B}, \mathrm{G}, \mathrm{H})]$, and cervical nerves [Fig. $5(\mathrm{~K})]$, and in the abdomen the proximal part of the abdominal nerve (results not shown). Furthermore, we found strong labeling in the region of the cervical connective [Fig. $5(\mathrm{C}, \mathrm{I})]$, the terminals of labial, pharyngeal, and accessory pharyngeal nerves in the subesophageal ganglion [Fig. $5(\mathrm{~K})$ ] and of the terminals of the leg, wing, haltere, and abdominal nerves in the thoracic ganglion [Fig. 5(C,H,K)], respectively. In contrast, no labeling was found in the region of the nerve terminals of the antennal nerve in the antennal lobe [Fig. 4(D)]. A specific labeling, although weaker and more dispersed, was found in the neuropil region of the brain [Fig. $5(\mathrm{C}, \mathrm{H}, \mathrm{I}, \mathrm{K})]$ and the neuropil region of the thoracic ganglion [Fig. 5(C,I,K)]. In the genital tract, oviduct [Fig. 5(M)] and uterus [Fig. 5(M,O)] were clearly labeled. The ovaries were not labeled [Fig. 5(O)].

All binding sites were competable with either nonradioactive sDUP99B or $\mathrm{SSP}$. Binding sites described for sDUP99B in the peripheral nervous system were also labeled by ${ }^{125} \mathrm{I}-\mathrm{YsSP}_{\mathrm{s}}$. Binding of synthetic sex peptide to the uterus, oviduct, cervical connective, and to the above-mentioned parts of the subesopha- 


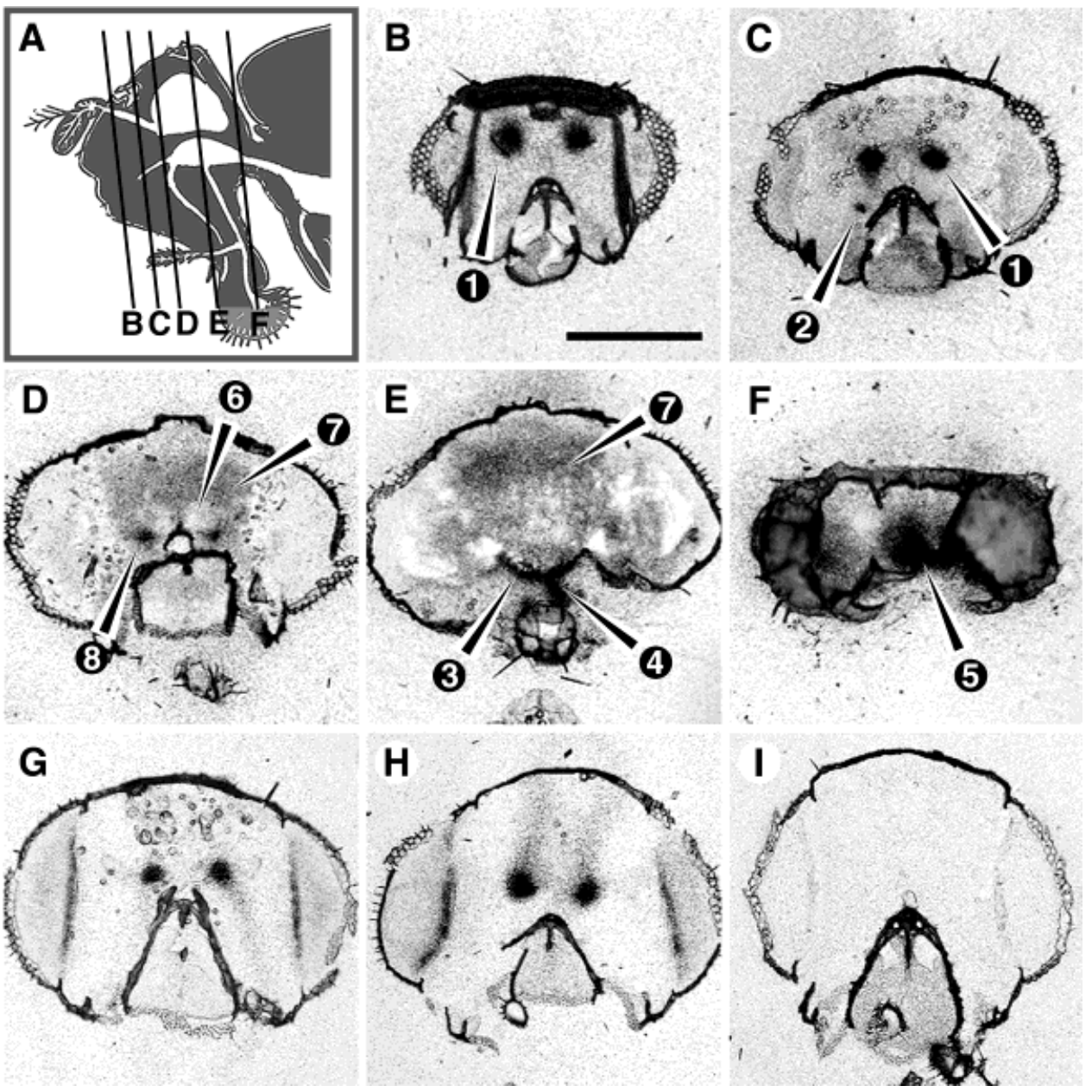

Figure $4{ }^{125}$ I-sDUP99B binds to peripheral nerves and the cervical connective in Drosophila heads. Binding of ${ }^{125}$ I-sDUP99B to frontal head sections of 5-day-old, sexually mature virgin (B-F) or mated (G) D. melanogaster females, D. melanogaster male (H), or virgin D. funebris (I) female. (A) Scheme of section planes for figures (B-F). (B-F) Autoradiographs of sectioning planes as indicated in (A). Arrowheads point toward the antennal nerve (1), pharyngeal nerve (2), periphery of subesophageal ganglion (3), branching labial nerve (4), cervical connective (5), antennal lobe (6, not labeled), brain (7, weakly labeled), and the antennal machanosensory center (8). The dark structure at the top of the head section in (B) is a chitinous structure of the head capsule. Scale bar $=300 \mu \mathrm{m}$.

geal and thoracic ganglia, however, was sometimes masked by the high background of the surrounding tissues. The only unspecific binding was found in the optical tracts as shown in Figures $2(\mathrm{G}-\mathrm{K})$ and $5(\mathrm{D}, \mathrm{E})$. Besides the tissues mentioned above, no other organs were found to be labeled in sexually mature, virgin females.

\section{Binding in Mated Females, Males, and D. funebris Females}

Both injection of SP into the abdomen of virgin females and ectopic expression of the SP gene in the fat body of virgins elicit the postmating responses (Aigaki et al., 1991; Schmidt et al., 1993). Hence, binding of SP to target sites in mated females could change the availability of binding sites for the radioligand on tissue sections. Differences between virgin and mated D. melanogaster females, however, were not found, neither in the labeling pattern nor in the intensity of labeling [Fig. 4(G) for the antennal nerve of a mated female, and results not shown]. This suggests that even small amounts of peptide are sufficient to induce the postmating responses and that the binding sites are not yet saturated in the mated females. 

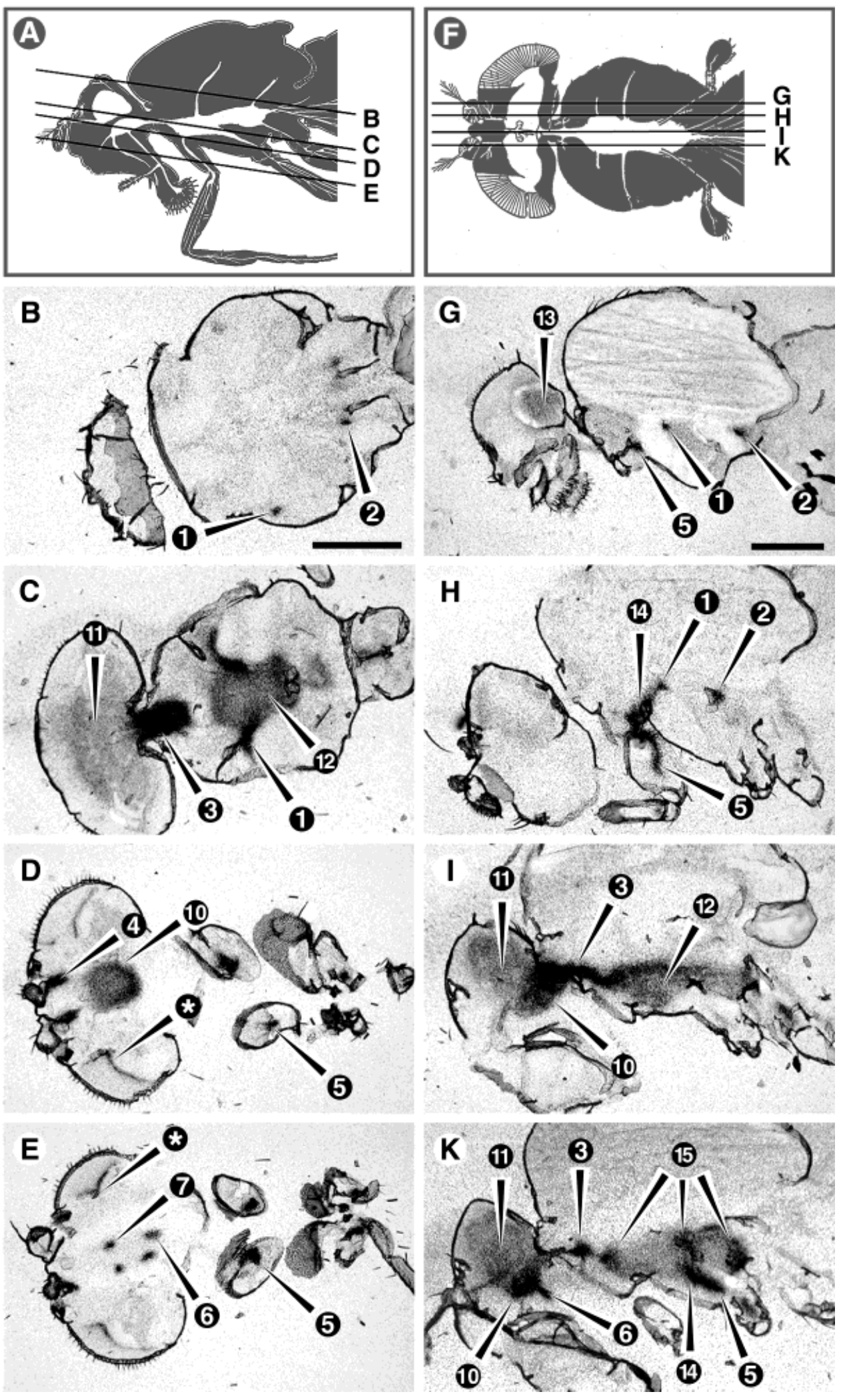

Figure 5 (Continued) 

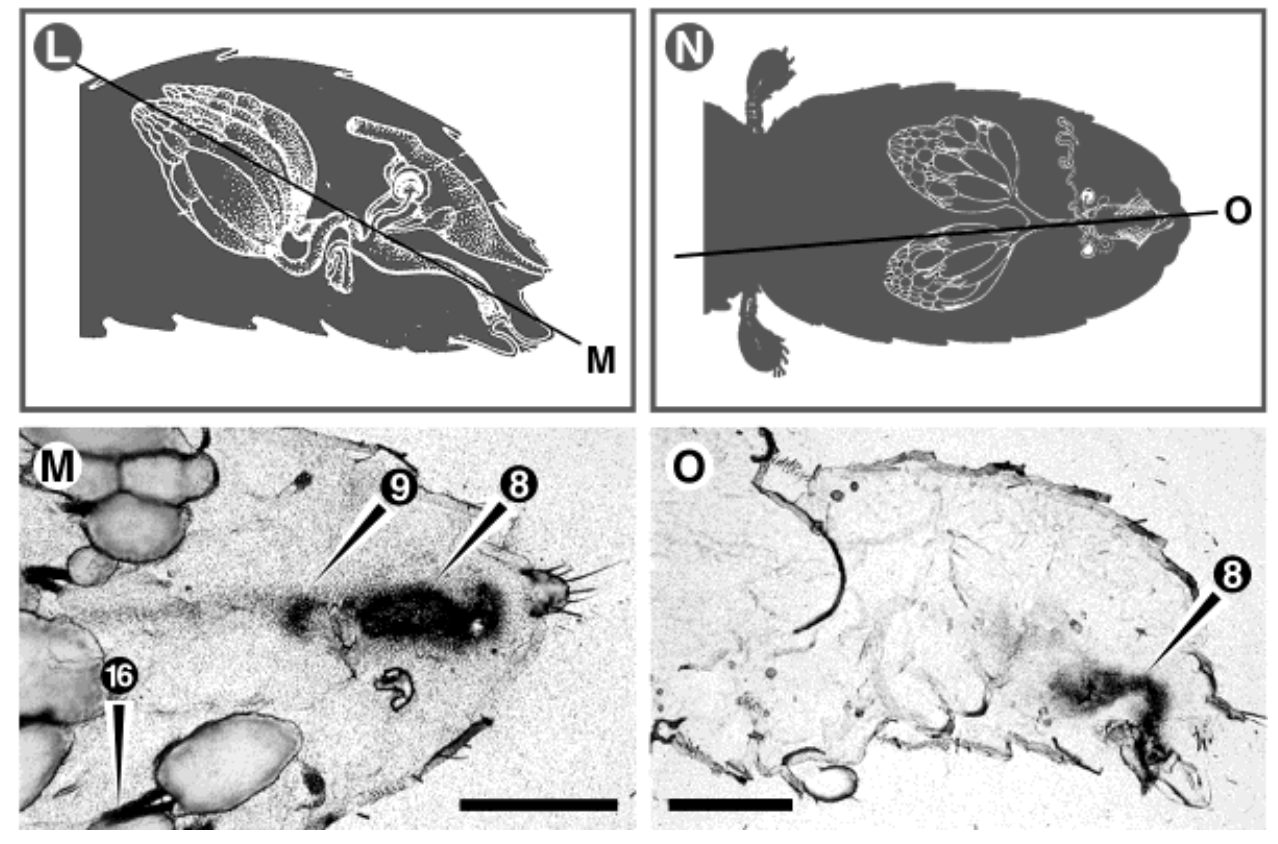

Figure $5{ }^{125}$ I-sDUP99B binds to peripheral nerves, the cervical connective, and parts of the subesophageal and thoracic ganglion. (A,F,L,N) Schematic of the section planes through 5-day-old, sexually mature virgin $D$. melanogaster females. (B-E) and $(\mathrm{G}-\mathrm{K})$ Sections through heads and thoraces; (M,O) sections through abdomina. Arrowheads point toward the wing nerve (1), haltere nerve (2), cervical connective (3), antennal nerve (4), leg nerve (5), labial nerve (6), accessory pharyngeal nerve (7), uterus (8), oviduct (9), subesophageal ganglion (10), brain (11), thoracic ganglion (12), optical lobe (13), leg nerve entry in the thoracic ganglion (14), sensory projections of leg, wing, and abdominal nerves in the ventral nerve cord (ventral ganglion, thoracico-abdominal ganglion; 15), chorionic appendage of an egg (16, not labeled). Unspecific binding, which was seen only in the optical tracts, is marked with a star. Scale bars $=300 \mu \mathrm{m}$.

This view is supported by earlier findings. Transfer of both peptides to female genital tracts could be demonstrated immediately after mating (Aigaki et al., 1991; Soller, M., Kubli, E., unpublished results). Peptide concentrations in the hemolymph of mated females, however, were below detection levels. Furthermore, the presence of SP after ubiquitous ectopic expression in transgenic females could not be demonstrated, although both postmating responses were elicited after heat induction of the transgene (Aigaki et al., 1991). Presence of SP in the hemolymph is clearly detectable, however, when expressed from a constitutively active yolk protein promoter, likely because of strong transcriptional activity from this promoter (data not shown).

Sex-peptide pheromones are synthesized in the accessory glands for the Ductus ejaculatorius, respectively, and leave the male body with the seminal fluid. Cryostat tissue sections of inexperienced D. melanogaster males incubated with ${ }^{125} \mathrm{I}$-sDUP99B showed specific labeling of the antennal nerve, indistinguishable from the binding in virgin females [Fig. 4(H)].
Also, with the exception of the thoracic ganglion where binding was less prominent in the male (data not shown), the binding patterns of both sex-peptide pheromones in other body parts were very similar in both sexes. In the male, however, no in vivo function is known for sex-peptide pheromones.

Females of $D$. funebris, a distantly related species not responding to D. melanogaster SP injections (Chen et al., 1988), did not show labeling of any tissues as exemplified in Figure 4(I) for the antennal nerve.

\section{Binding Sites Appear at Specific Developmental Stages}

The maturation of the SP-response system in females occurs in specific steps (Moshitzky et al., 1996). Ovulation can only be induced by SP injection about $38 \mathrm{~h}$ after eclosion, although mature eggs are present and eventually laid after about 1 day. Sex-peptide injection fully reduces receptivity only $72 \mathrm{~h}$ after eclosion (Fleischmann, 1991). We therefore investigated 


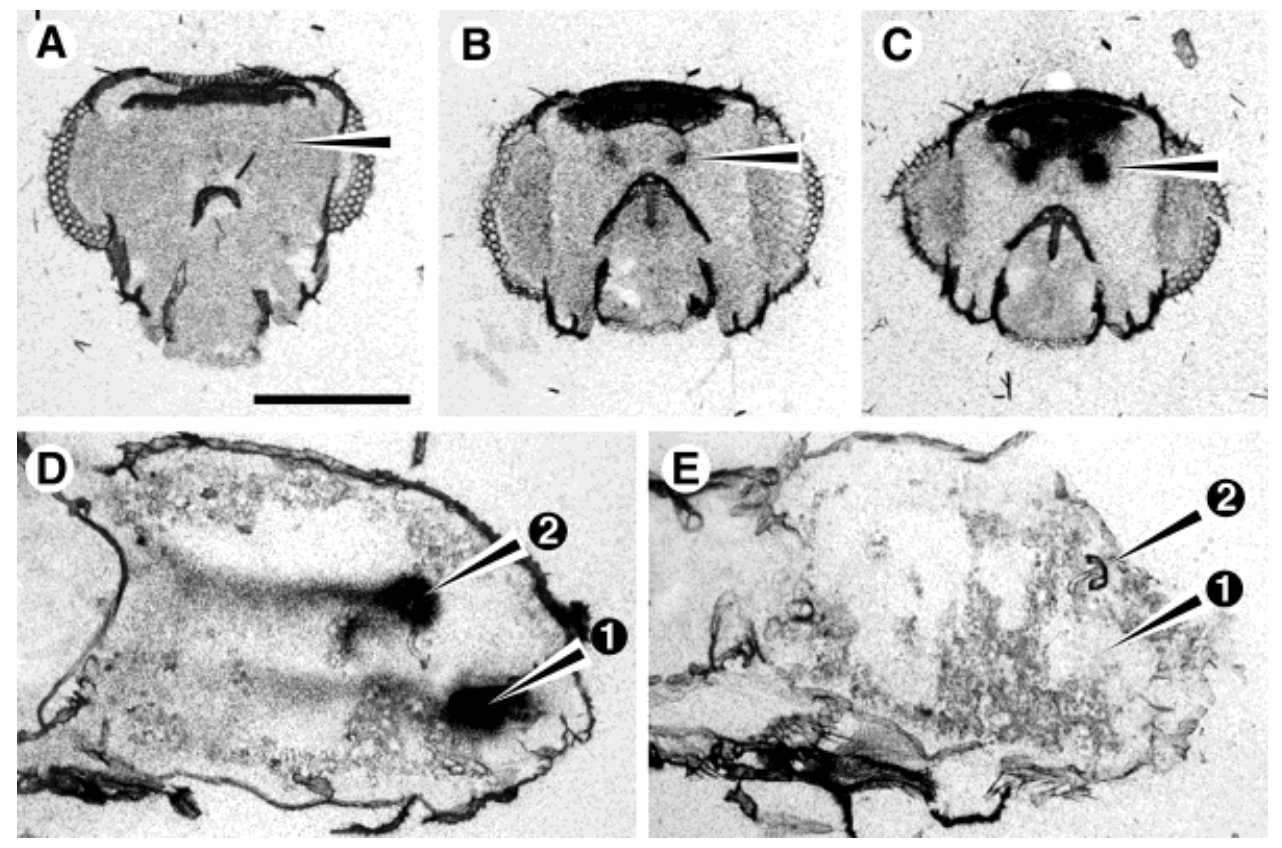

Figure $6{ }^{125}$ I-sDUP99B binding sites are established through sexual maturation. Binding of ${ }^{125}$ I-sDUP99B to frontal sections of 2- (A), 11- (B), and 36-h (C)-old virgin D. melanogaster females and a final pupal stage P14 (D,E) are shown. Arrowheads point toward the antennal nerve in $(A-C)$ and to the genital tract (1) and the spermathecae (2) in (D) and (E), respectively. The dark structure at the top of the head sections in $(\mathrm{A}-\mathrm{C})$ is a chitinous structure of the head capsule. Note that ${ }^{125}$ I-sDUP99B binding sites are only fully established about $36 \mathrm{~h}$ after eclosion from the pupae. (E) Competition of ${ }^{125}$ I-DUP99B binding with a 250 -fold excess of sDUP99B. Labeling on the genital tract and spermathecae is absent. Scale bar $=300 \mu \mathrm{m}$.

whether the development of the labeling patterns of the nervous system and genital tract correlates with the observed steps of maturation of the SP-response cascade.

Labeling intensities at the antennal nerve were quantified 2, 11, and $36 \mathrm{~h}$ after eclosion. No labeling was observed immediately after eclosion [Fig. 6(A)], whereas $11 \mathrm{~h}$ after eclosion half of the maximal intensity, as measured in 5-day-old females, was established [Fig. 6(B)]. After $36 \mathrm{~h}$ the labeling intensity was the same as in 5-day-old females [Fig. $6(\mathrm{C})]$. The complete number of axons in the antennal nerve, however, may be present before eclosion (Salecker and Malun, 1999; Stocker, R., unpublished results). Hence, the absence of antennal nerve labeling in females immediately after eclosion may be due to the lack of binding sites.

Some neuronal binding sites in other parts of the fly are visible immediately after eclosion. The complete binding pattern, however, is only fully established at about $36 \mathrm{~h}$ after eclosion. Binding sites in the genital tract, but not in the nervous system, can already be identified in the final pupal stage P14 [Fig.
6(D)]. At the same stage, specific binding was also found in the region of the spermathecae [Fig. 6(D)]. This latter labeling, however, disappears 1 day after eclosion. It is considered to be specific, because it can be competed with a 250 -fold excess of nonradioactive peptide [Fig. 6(E)].

\section{DISCUSSION}

\section{Specificity of Binding Sites}

Our approach directly detects and locates proteins interacting with the labeled ligand. The following arguments support our conclusion that we have localized specific binding sites and thus the expression of putative receptors for DUP99B and SP. (1) The iodinated sex peptides used as probes are biologically active. (2) The binding patterns are reproducible, and labeling disappears in competition experiments with nonradioactive peptides. (3) All binding sites are competable only with biologically active fragments of sDUP99B or sSP, delineating the activity of the pep- 
tides to the second exon. This finding is in agreement with the analysis of structure-function relationships showing that the same C-terminal fragment is essential for eliciting the two postmating responses (Schmidt et al., 1993). (4) The determined dissociation constant $K_{\mathrm{d}}$ of $6.4 \times 10^{-9} \mathrm{M}$ is in the range of known values for hormone-receptor interactions (Merckaert and Vandesande, 1996; Cicutti et al., 1999). (5) Binding sites in the adult female are established during sexual maturation and correlate with the establishment of the behavioral response (Fleischmann, 1991; Moshitzky et al., 1996). (6) No binding was observed in $D$. funebris females, a distantly related, SP-insensitive Drosophila species.

\section{Sex-Peptide Pheromone Target Sites}

Prominent labeling in clearly defined regions was found in parts of the nervous system and the genital tract. In the nervous system regions of strong (cervical connective, peripheral nerves, and their terminals in the subesophageal and thoracic ganglia) and weak labeling (neuropil of brain and neuropil of thoracic ganglion) could be observed (Fig. 7). In 5-day-old virgins, strong labeling is found in regions where the neurons are bundled into tight tracts (e.g., the cervical connective). Weaker and more dispersed labeling is found in the neuropil of the brain and the thoracic ganglion. The following findings, however, suggest that the radiolabeled ligands bind to specific sites in the nervous system. (1) The stomatogastric nervous system and the antennal lobes are not labeled. (2) In the subesophageal and thoracic ganglia strong labeling was also observed in regions of the cell bodies, the cortex. (3) Only the proximal parts of the peripheral nerves are labeled. (4) The labeling patterns change during development, and the intensity is not always correlated with tight axon tracts. Specific labeling patterns and developmental changes have also been described in mammals. For example, in mice, receptors for the abundant and widely expressed neuropeptide $\mathrm{Y}$ have been localized in the peripheral and CNS. different classes of receptors seem to be involved in different neuropeptide $\mathrm{Y}$ functions, and, furthermore, they seem to play an important role in nervous system development (Naveilhan et al., 1998).

In contrast to other neuronal projections, the terminals of the olfactory tracts of the antennal and labial nerves in the brain were not labeled. In all peripheral nerves, the labeling was never found to extend to distal parts, for example, in the antennal nerve it was restricted to the region between the antennal lobes and the basis of the antenna. Labeling was never found in

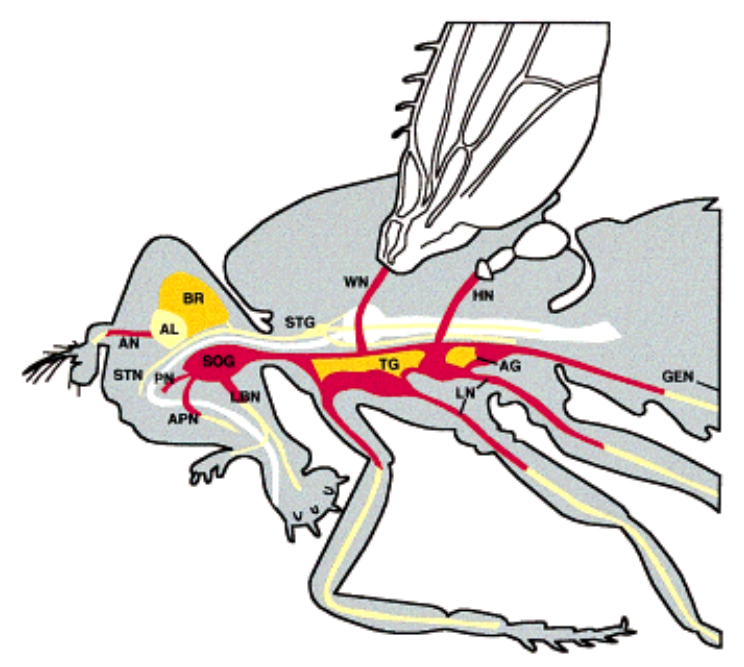

Figure 7 Schematic overview of sex-peptide pheromone binding sites to the nervous system (excluding the abdomen) of sexually mature virgin $D$. melanogaster females. Strong labeling of the nervous system is indicated in red, weak labeling in orange, and unlabeled parts of the nervous system are shown in yellow. The digestive system is shown in white. AG, abdominal ganglion; AL, antennal lobe; AN, antennal nerve; APN, accessory pharyngeal nerve; BR, brain; GEN, genital nerve; HN, haltere nerve; LBN, labial nerve; LN, leg nerve; PN, pharyngeal nerve; STG, stomatogastric ganglion; STN, stomatogastric nerve; SOG, subesophageal ganglion; TG, thoracic ganglion; WN, wing nerve.

the antenna or in the region of the sensilla. Labeling of the terminal abdominal nerve was found as far as the second sternite, in the leg nerves to the femur, and in the haltere nerves to the scabellum.

The labeling pattern along the peripheral nerves might indicate a common function of the labeled nerves. The label may be bound to sensory afferents or glial cells. Localization in sensory fibers is suggested by the fact that all labeled nerves contain afferents, and some do not contain motor efferents (e.g., the haltere nerve; Hertweck, 1931; Power, 1948). Also, the strongly labeled antennal nerve is almost fully afferent. Less than 10 of the 1800 axons present at the site of entry into the head capsule (Stocker, 1979) may be efferents, because the only peripheral targets of the antennal nerve are two small antennal muscles (Miller, 1950). Hence, binding of the label on motor axons would be barely visible. Furthermore, in other mixed nerves, such as wing, leg, labial, and pharyngeal nerves, the simultaneous labeling of CNS areas adjacent to the entry of the nerves [Fig. $5(\mathrm{H}, \mathrm{K})$ ] suggests the marking of afferent projections. With respect to the antennal nerve, labeling is 
seen only in the mechanosensory antennal center, but not in the olfactory antennal lobe [Fig. 4(D)]. The strong labeling of the cervical connective, however, suggests that elements apart from afferents may be involved as well, because the cervical connective contains only a minor subpopulation of afferents, originating predominantly from wings and halteres (Ghysen, 1978; Palka et al., 1979; Ghysen et al., 1983).

Alternatively, sex-peptide pheromones may bind to glial cells in the peripheral nerves or to both glia and afferents. The resolution of ${ }^{125}$ I autoradiography does not allow us to discriminate between these possibilities. Recent work with glia has revised the view of a mainly passive role of these cells (Barres, 1991; Ullian and Barres, 1998). Glia cells contain voltagegated and ligand-dependent ion channels (Barres et al., 1989, 1990). Modulation of synaptic efficacy and synaptic depression by glial cells at the frog neuromuscular junction has been demonstrated (Robitaille, 1998). Hence, glial cells contain the machinery to receive and transmit humoral signals. Sex-peptide pheromone targets are accessible via the hemolymph, inasmuch as injected peptides elicit full biological activity. Thus, it is conceivable that sex-peptide pheromone receptors might be located on glial cells. Alternatively, glial cells may express a transport system allowing the passage of the peptides through the blood-brain barrier. Transport of neuropeptides along neurons (e.g., in the brain-retrocerebral neuroendocrine complex), is well known and has been studied in detail in many insect species (for review see Nijhout, 1994).

In our experiments, labeling was also found in the genital tract, the site of first contact of the seminal fluid with the female tissues after copulation. Monsma et al. (1990) showed that in the D. melanogaster female, accessory gland proteins enter the hemolymph, probably mediated by a transport system. Thus, the identified binding sites in the genital tract could indicate the presence of a transporter. In vertebrates, however, the same hormone receptors have been localized in neuronal tissue and in the uterus (Quinones-Jenab et al., 1997). Hence, the genital tract of D. melanogaster females may posses receptors of its own.

Both peptides bind also to male tissue sections [Fig. 4(H), and results not shown]. In the CNS and the peripheral nervous system, the labeling pattern corresponds more or less to the female pattern, with the exception of the thoracic ganglion. Whether these binding sites are functional is not known. In vertebrates, expression of estrogen and testosterone receptors, respectively, are also found in the opposite sex. Both types of receptors are necessary ontogenetically and physiologically in both sexes (Cooke et al., 1991; Kelly, 1991; MacLusky and Naftolin, 1981).

\section{Sex-Peptide Pheromone Response Cascade}

The CA is a likely target of SP because (1) SP stimulates $\mathrm{JH}$ synthesis in this gland in vitro (Moshitzky et al., 1996) and (2) both, SP and JH, increase egg production (Soller et al., 1999). In our experiments, however, we did not find binding of DUP99B to the CA, although the neighboring cervical connective is strongly labeled [Fig. 5(C,I)]. In vitro studies with $\mathrm{N}$-terminal sSP fragments in the CA assay indicate that the highly conserved $\mathrm{N}$-term of SP is the functional part inducing $\mathrm{JH}$-synthesis (Fan et al., 2000). This is confirmed by the finding that DUP99B has no effect on JH synthesis in the CA assay (Fan et al., 2000; Moshitzky, P., Applebaum, S., Kubli, E., unpublished results). Sex-peptide and DUP99B are highly homologous in their C-terminal parts, but differ, however, in their N-terminal parts. The lack of labeling of the CA by radiolabeled DUP99B is therefore consistent with these findings. The lack of labeling by ${ }^{125} \mathrm{I}$-SP might be due either to the elimination of binding capability by the introduction of a tyrosine residue at the $\mathrm{N}$-term or to the difficulty of detecting a weak signal. Because JH neither elicits egg laying nor reduction of receptivity, SP must have other targets.

Experiments with gynandromorphs have shown that the focus for egg laying is localized in the thoracic ganglion (Szabad and Fajszi, 1982), whereas the focus for receptivity is localized in the dorsal-anterior part of the brain (Tompkins and Hall, 1983). These findings suggest that the postmating responses elicited by the two sex-peptide pheromones, SP and DUP99B, are controlled by two different centers. The focus for receptivity contains cell bodies with axons projecting into the mushroom bodies, brain structures involved in learning, memory, and sexual orientation (Heisenberg, 1994; Ferveur et al., 1995; O'Dell et al., 1995). Chemical deletion of the mushroom bodies, however, does not affect the SP response (Fleischmann, 1997). This finding is consistent with the lack of labeling of these brain structures [Fig. 4(E)]. Indirect evidence, however, indicates that only one type of molecular receptor is present for the two postmating responses (Schmidt et al., 1993; Kubli, 1996; Nakayama et al., 1997). Thus, the same type of molecular receptor is presumably expressed at different sites of the nervous system.

Virgin and mated females confronted with the 
same stimulus, courting males, respond with opposite behaviors. How do the sex-peptide pheromones induce this behavioral switch in females? The foci for female postmating behaviors, as mapped by gynandromorph experiments, probably represent neuronal circuits involved in the integration of sensory input and/or the execution of behaviors related to the sexual state. In rodents, sites of steroid sex-hormone action, or pheromone-induced alterations in the nervous system, have been mapped to discrete neuronal circuits. They can induce behavioral or physiological changes due to their neuroendocrine or neuronal nature (Mani et al., 1995; for review see Keverne, 1983, 1999; McCarthy and Albrecht, 1996; McCarthy and Pfaus, 1996). At present we can not exclude that in Drosophila SP pheromones act on discrete neuronal circuits. Binding to proximal parts of peripheral nerves, however, may indicate that they act differently. For example, they could modulate received sensory input globally, rather than act at the foci mapped by gynandromorph experiments, where we did not detect increased labeling. In this way the same stimuli could result in an altered behavioral response of the female, depending on the presence or absence of sex peptide pheromones (e.g., engagement in mating versus rejection of courting males in virgin and mated females, respectively). This view is supported by an observation involving a behavioral change elicited by mating in the medfly Ceratitis capitata (Jang, 1995). Although virgin females prefer male pheromones, after mating they are attracted by the scent of mature guava fruit. This odor preference switch can be elicited by injection of accessory gland extracts prepared from $C$. capitata males into virgin females. The responsible substance may be an SP-like molecule modulating the olfactory input in mated females. Whether SP pheromones or functionally related peptides modify sensory input or act on discrete neuronal circuits, however, awaits further molecular and neuronal characterization of the involved signaling mechanisms.

The authors thank Richard Burke for critically reading the manuscript, Peter Gehrig for mass spectroscopy, Walter Lichtensteiger for valuable discussions about binding assays, Martin Heisenberg for fly collars, and Yves Choffat and Thomas Fritschi for art work, technical advice, and various support.

\section{REFERENCES}

Aigaki T, Fleischmann I, Chen PS, Kubli E. 1991. Ectopic expression of sex peptide alters reproductive behavior of female D. melanogaster. Neuron 7:557-563.
Barres BA. 1991. New roles for glia. J Neurosci 11:36853694.

Barres BA, Chun LL, Corey DP. 1989. Glial and neuronal forms of the voltage-dependent sodium channel: characteristics and celltype distribution. Neuron 2:1375-1388.

Barres BA, Koroshetz WJ, Swartz KJ, Chun LL, Corey DP. 1990. Ion channel expression by white matter glia: the O-2A glial progenitor cell. Neuron 4:507-524.

Boulétreau-Merle J. 1976. Destruction of the pars intercerebralis in Drosophila melanogaster: effect on the fecundity and the stimulation through copulation. J Insect Physiol 2:933-940.

Chapman T, Choffat Y, Lucas WE, Kubli E, Partridge L. 1996. Lack of response to sex-peptide results in increased cost of mating in dunce Drosophila melanogaster females. J Insect Physiol 42:1007-1015.

Chen CN, Denome S, Davis RL. 1986. Molecular analysis of cDNA clones and the corresponding genomic coding sequences of the Drosophila dunce+ gene, the structural gene for cAMP phosphodiesterase. Proc Natl Acad Sci USA 83:9313-9317.

Chen PS, Stumm-Zollinger E, Aigaki T, Balmer J, Bienz M, Böhlen P. 1988. A male accessory gland peptide that regulates reproductive behavior of female $D$. melanogaster. Cell 54:291-298.

Cicutti NJ, Smyth CE, Rosaeg OP, Wilkinson M. 1999. Oxytocin receptor binding in rat and human heart. Can J Cardiol 15:1267-1273.

Cooke PS, Young P, Hess RA, Cunha GR. 1991. Estrogen receptor expression in developing epididymis, efferent ductules, and other male reproductive organs. Endocrinology 128:2874-2879.

Dudai Y, Amsterdam A. 1977. Nicotinic receptors in the brain of Drosophila melanogaster demonstrated by autoradiography with $\left[{ }^{125} \mathrm{I}\right]$ alpha-bungarotoxin. Brain Res 130:551-555.

Eberhard WG. 1996. Female Control: sexual selection by cryptic female choice. Princeton: Princeton University Press. p 501.

Fan Y, Rafaeli A, Moshitzky P, Kubli E, Choffat Y, Applebaum SW. 2000. Common functional elements of Drosophila melanogaster seminal peptides involved in reproduction of Drosophila melanogaster and Helicoverpa armigera females. Insect Biochem Mol Biol, (In press).

Ferveur JF, Störtkuhl KF, Stocker RF, Greenspan RJ. 1995. Genetic feminization of brain structures and changed sexual orientation in male Drosophila. Science 267:902 905.

Fleischmann I. 1991. Die Reifung des weiblichen Sexualverhaltens der Taufliege Drosophila melanogaster. Diplomarbeit. Universität Zürich.

Fleischmann I. 1997. Female reproductive behavior of Drosophila modulated by a male pheromone: the nervous system as a site of sex-peptide action. Dissertation Universität Zürich.

Ghysen A. 1978. Sensory neurones recognise defined path- 
ways in Drosophila central nervous system. Nature 274: 869-872.

Ghysen A, Janson R, Santamaria P. 1983. Segmental determination of sensory neurons in Drosophila. Dev Biol 99:7-26.

Gillott C. 1988. Arthropoda-Insecta. In: Adyodi KG Adyodi RG, editors. Reproductive biology of invertebrates. Chichester: Wiley and Sons. p 319-471.

Hafen E, Levine M. 1986. Localization of RNAs by in situ hybridization. In: Roberts DB, editor. Drosophila-a practical approach. Oxford: IRL Press. p 139-157.

Hauck K. 1995. Biochemische und molekularbiologische Charakterisierung eines männlichen "Kopffaktors" (MHF) aus Drosophila melanogaster. Diplomarbeit. Universität Zürich.

Heisenberg M. 1994. Central brain function in insects: genetic studies on the mushroom bodies and central complex in Drosophila. In: Schildberger K Elsner N, editors. Neural basis of behavioural adaptions. Stuttgart: Gustav Fischer Verlag. p 61-79.

Herndon LA, Wolfner MF. 1995. A Drosophila seminal fluid protein, Acp26Aa, stimulates egg laying in females for 1 day after mating. Proc Natl Acad Sci USA 92: 10114-10118.

Hertweck H. 1931. Anatomie und Variabilität des Nervensystems und der Sinnesorgane von Drosophila melanogaster (Meigen). Zwiss Zool 139:559-663.

Hilfiker A. 1990. Genetic and developmental analysis of virilizer, a gene involved in sex determination and dosage compensation of Drosophila melanogaster. Dissertation. Universität Zürich.

Jang EB. 1995. Effects of mating and accessory gland injections on olfactory-mediated behavior in the female mediterranean fruit fly, Ceratitis capitata. J Insect Physiol 4:705-710.

Karlson P, Lüscher M. 1959. "Pheromones": a new term for a class of biologically active substances. Nature 183:5556.

Kelly DD. 1991. Sexual differentiation of the nervous system. In: Kandel ER, Schwartz JH, Jessel TM, editors. Principles of neural science. Amsterdam: Elsevier. $\mathrm{p}$ 959-973.

Keverne EB. 1983. Pheromonal influences on the endocrine regulation of reproduction. Trends Neurosci 6:381-384.

Keverne EB. 1999. The vomeronasal organ. Science 286: 716-720.

Kubli E. 1996. The Drosophila sex-peptide: a peptide pheromone involved in reproduction. Adv Dev Biochem 4:99-128.

Lichtensteiger W, Hanimann B, Siegrist W, Eberle AN. 1996. Region- and stage-specific patterns of melanocortin receptor ontogeny in rat central nervous system, cranial nerve ganglia and sympathetic ganglia. Brain Res Dev Brain Res 91:93-110.

MacLusky NJ, Naftolin F. 1981. Sexual differentiation of the central nervous system. Science 211:1294-1302.

Mani SK, Allen JM, Clark JH, Blaustein JD, O’Malley BW.
1995. Convergent pathways for steroid hormone- and neurotransmitter-induced rat sexual behavior. Science 265:1246-1249.

McCarthy MM, Albrecht ED. 1996. Steroid regulation of sexual behaviour. Trends Endocrin Metab 7:324-327.

McCarthy MM, Pfaus PG. 1996. Steroid modulation of neurotransmitter function to alter female reproductive behaviour. Trends Endocrin Metab 7:327-333.

Merckaert J, Vandesande F. 1996. Autoradiographic localization of receptors for neuropeptide Y (NPY) in the brain of broiler and leghorn chickens (Gallus domesticus). J Chem Neuroanat 12:123-134.

Miller A. 1950. The internal anatomy and histology of the imago of Drosophila melanogaster. In: Demerec M, editor. The biology of Drosophila. London: John Wiley \& Sons. p 420-534.

Monsma SA, Harada HA, Wolfner MF. 1990. Synthesis of two Drosophila male accessory gland proteins and their fate after transfer to the female during mating. Dev Biol 142:465-475.

Moshitzky P, Fleischmann I, Chaimov N, Saudan P, Klauser S, Kubli E, Applebaum SW. 1996. Sex-peptide activates juvenile hormone biosynthesis in the Drosophila melanogaster corpus allatum. Arch Insect Biochem Physiol 32:363-374.

Nakayama S, Kaiser K, Aigaki T. 1997. Ectopic expression of sex-peptide in a variety of tissues in Drosophila females using the P[GAL4] enhancer-trap system. Mol Gen Genet 254:449-455.

Naveilhan P, Neveu I, Arenas E, Ernfors P. 1998. Complementary and overlapping expression of $\mathrm{Y} 1, \mathrm{Y} 2$ and $\mathrm{Y} 5$ receptors in the developing and adult mouse nervous system. Neuroscience 87:289-302.

Nighorn A, Healy MJ, Davis RL. 1991. The cyclic AMP phosphodiesterase encoded by the Drosophila dunce gene is concentrated in the mushroom body neuropil. Neuron 6:455-467.

Nijhout HF. 1994. Insect hormones. Princeton: Princeton University Press.

O’Dell KM, Armstrong JD, Yang MY, Kaiser K. 1995. Functional dissection of the Drosophila mushroom bodies by selective feminization of genetically defined subcompartments. Neuron 15:55-61.

Palka J, Lawrence PA, Hart HS. 1979. Neural projection patterns from homeotic tissue of Drosophila studied in bithorax mutants and mosaics. Dev Biol 69:549-575.

Power ME. 1948. The thoracico-abdominal nervous system of an adult insect, Drosophila melanogaster. J Comp Neurol 88:347-391.

Quinones-Jenab V, Jenab S, Ogawa S, Adan RA, Burbach JP, Pfaff DW. 1997. Effects of estrogen on oxytocin receptor messenger ribonucleic acid expression in the uterus, pituitary, and forebrain of the female rat. Neuroendocrinology 65:9-17.

Robitaille R. 1998. Modulation of synaptic efficacy and synaptic depression by glial cells at the frog neuromuscular junction. Neuron 21:847-855. 
Salecker I, Malun D. 1999. Development of olfactory glomeruli. In: Hanson BS, editor. Insect olfaction. Berlin: Springer-Verlag. p 207-242.

Saudan P. 1993. Charakterisierung eines Drosophila Kopffaktors, der die Ovulation und Oviposition virgineller Weibchen stimuliert und deren Rezeptivität senkt. Diplomarbeit. Universität Zürich.

Schägger H, von Jagow G. 1987. Tricine-sodium dodecyl sulfate-polyacrylamide gel electrophoresis for the separation of proteins in the range from 1 to $100 \mathrm{kDa}$. Anal Biochem 166:368-379.

Schmidt T, Choffat Y, Klauser S, Kubli E. 1993. The Drosophila melanogaster sex-peptide: a molecular analysis of structure-function relationships. J Insect Physiol 39:361-368.

Soller M, Bownes M, Kubli E. 1997. Mating and sexpeptide stimulate the accumulation of yolk in oocytes of Drosophila melanogaster. Eur J Biochem 243:732-738.
Soller M, Bownes M, Kubli E. 1999. Control of oocyte maturation in sexually mature Drosophila females. Dev Biol 208:337-351.

Stocker RF. 1979. Fine structural comparison of the antennal nerve in the homeotic mutant Antennapedia with the wild-type antennal and second leg nerves of Drosophila melanogaster. J Morphol 160:209-222.

Szabad J, Fajszi C. 1982. Control of female reproduction in Drosophila: genetic dissection using gynandromorphs. Genetics 100:61-78.

Tompkins L, Hall JC. 1983. Identification of brain sites controlling female receptivity in mosaics of Drosophila melanogaster. Genetics 103:179-195.

Ullian EM, Barres BA. 1998. The Schwann song of the glia-less synapse. Neuron 21:651-652.

Wolfner MF. 1997. Tokens of love: functions and regulation of Drosophila male accessory gland products. Insect Biochem Mol Biol 27:179-192. 